\title{
Long-term treatment with anakinra and canakinumab resolves patellar subchondral erosion in neonatal-onset multisystem inflammatory disease
}

\author{
A. Leone ${ }^{1,2}$, R. Manna ${ }^{2,3}$, E. Verrecchia ${ }^{3}$, M. Cipullo ${ }^{4}$, D. Rigante ${ }^{4,2}$ \\ 'Institute of Radiology, Fondazione Policlinico Universitario A. Gemelli IRCCS, Rome, Italy; \\ ${ }^{2}$ Università Cattolica Sacro Cuore, Rome, Italy; ${ }^{3}$ Institute of Internal Medicine, Fondazione Policlinico \\ Universitario IRCCS, Rome, Italy; ${ }^{4}$ Institute of Pediatrics, Fondazione Policlinico Universitario \\ IRCCS, Rome, Italy
}

\begin{abstract}
Tise o the Editor:

The pathogenetic mechanisms behind skeletal and cartilaginous manifestations of neonatal-onset multisystem inflammatory disease (NOMID) are little known. It is a rare, inherited, autoinflammatory disease defined by fever, urticaria-like rash, ocular signs of different severity, chronic meningitis and a peculiar osteoarthropathy, characterized by irregular femoral enlargement and bizarre patellar protrusion $(1,2)$. All NOMID manifestations start soon after birth and are widely thought to be driven by the uncontrolled production of interleukin (IL)-1 $(3,4)$. Sixty per cent of NOMID patients carry gain-offunction missense mutations in the NLRP3 gene, which encodes for cryopyrin, an apoptosis regulation protein controlling IL-1 biosynthesis, expressed primarily in phagocytic cells and chondrocytes: cryopyrin-associated periodic syndrome is the name given to all genetic disorders caused by aberrant cryopyrin function and oversecretion of IL-1 (5). NOMID is the most severe subphenotype of cryopyrin-associated periodic syndrome, displaying a relevant risk of joint contracture, limb length discrepancy and early degenerative arthropathy, mostly involving knees and frankly leading to protrusion of the patella (6). Very few data providing the long-term response to treatment with IL-1 inhibitors are available for NOMID and no information is available to define the response of skeletal and cartilaginous manifestations to this specific treatment (7).
\end{abstract}

We have managed a Caucasian man with
NOMID, who is now 20-year-old, in whom the disease started at birth, when protruding patellae were also noted, but was diagnosed at 3 years (by the demonstration of the missense mutation $\mathrm{T} 1718 \mathrm{C}$ in the NLRP3 gene), when the boy was unable to walk without aid. An initial treatment with the tumour necrosis factor inhibitor etanercept $(0.4 \mathrm{mg} / \mathrm{kg}$ twice a week subcutaneously) was started at 4 years and stopped after one year because unsuccessful (8). The IL-1 receptor antagonist anakinra was started at 7 years and kept for an overall period of 11 years, at a first dosage of $1 \mathrm{mg} / \mathrm{kg}$ of body weight/day subcutaneously, then changed to $2 \mathrm{mg} / \mathrm{kg} / \mathrm{day}$ (9). At the magnetic resonance imaging (MRI) of knees, performed at 13 years, we noticed a focal bone erosion on the posterior surface of both patellae combined with cartilage loss (Figure 1A and B): this was the first illustrated case of an evident patellar subchondral focal bone erosion in NOMID, notwithstanding IL-1 blockade (10). However, every clinical manifestation was highly responsive to anakinra, with the exception of bone abnormalities. A switch to therapy with the human anti-IL-1 monoclonal antibody canakinumab was decided at 17 years (300 mg subcutaneously every 3-4 weeks) and this therapy is still in course (11). At 20 years, a new MRI of knees showed the disappearance of the previously described osteochondral lesion in both patellae (Figure $1 \mathrm{C}$ and $\mathrm{D}$ ).

The complexity of treating patients with $\overline{\text { Corresponding author: }}$

Donato Rigante

Institute of Pediatrics,

Fondazione Policlinico Universitario A. Gemelli

Università Cattolica Sacro Cuore

Largo A. Gemelli, 8 - 00168 Rome, Italy

E-mail:drigante@gmail.com 

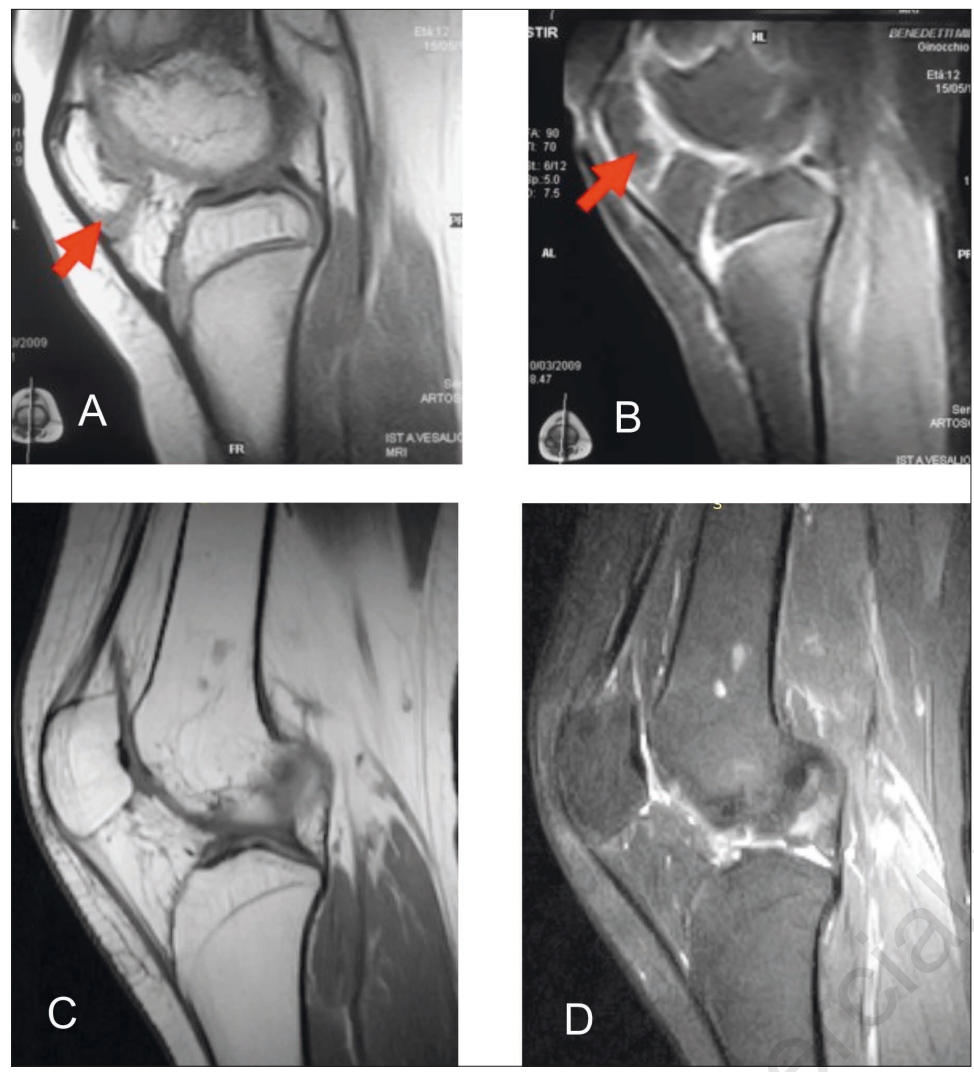

Figure 1 - A-D) Right knee magnetic resonance imaging. A) Sagittal T1-weighted and B) corresponding sagittal STIR images obtained at 13 years (after 6 years of treatment with anakinra) showing cartilage loss and subchondral erosion on the posterior surface of the patella (arrow in a and b) with no significant joint effusion. C) Sagittal T1weighted and D) corresponding sagittal STIR images obtained about seven years later (after 11 years of treatment with anakinra on the whole and 3 years of treatment with canakinumab) showing the disappearance of the previously reported erosion in the patella.

NOMID derives from the incomplete elucidation of the pathogenesis of its skeletal and cartilaginous manifestations: the repertoire of IL-1-mediated signalling systems are probably more complex at the bone level than in other body sites (12). In consideration of the pivotal role of IL-1 in the pathogenesis of NOMID, both anakinra and canakinumab have been approved for use in NOMID, with the aim of controlling symptoms, reducing biochemical activity markers, as well as improving patients' overall quality of life. Instruments to measure chronic damage in autoinflammatory diseases have been developed so far, but still await to be validated, also for NOMID which is characterized by the highest risk of progressive skeletal damage over time $(13,14)$. Bone abnormalities including the knees and patellae have recently been included in the diagnostic criteria of NOMID (15), but their improvement under IL-1 blockade has been little studied. Normally the hyaline cartilage on the back surface of the patella is thicker than in other sites of the body, and sometimes erosions can be disclosed during the course of various inflammatory diseases specifically involving this part of the body (16).

Our experience shows for the first time the resolution of a focal bone erosion in both patellae of a young patient with NOMID, after a longstanding treatment including first anakinra and then canakinumab. Prospective observational studies are needed to confirm long-term efficacy and sustained benefit from these drugs to encourage clinicians to use IL-1 antagonists as a very first-line therapy in NOMID to prevent the occurrence of irreversible bone changes.

Informed consent: Informed consent was obtained from the patient's parents and the patient himself to publish this article.

\section{REFERENCES}

1. Cantarini L, Lucherini OM, Frediani B, et al. Bridging the gap between the clinician and the patient with cryopyrin-associated periodic syndromes. Int $\mathrm{J}$ Immunopathol Pharmacol. 2011; 24: 827-36.

2. Rigante D, Vitale A, Lucherini OM, Cantarini. The hereditary autoinflammatory disorders uncovered. Autoimmun Rev. 2014; 13: 892-900.

3. Rigante D. A developing portrait of hereditary periodic fevers in childhood. Expert Opin Orphan Drugs. 2018; 6: 47-55.

4. Rigante D. New mosaic tiles in childhood hereditary autoinflammatory disorders. Immunol Lett. 2018; 193: 67-76.

5. Rigante D. A systematic approach to autoinflammatory syndromes: a spelling booklet for the beginner. Expert Rev Clin Immunol. 2017; 6: 571-97.

6. Torbiak RP, Dent PB, Cockshott WP. NOMID - a neonatal syndrome of multisystem inflammation. Skeletal Radiol. 1989; 18: 359-64.

7. Caso F, Cantarini L, Lucherini OM, et al. Working the endless puzzle of hereditary autoinflammatory disorders. Mod Rheumatol. 2014; 24 : 381-9. 
8. Federico G, Rigante D, Pugliese AL, et al. Etanercept induces improvement of arthropathy in chronic infantile neurological cutaneous articular (CINCA) syndrome. Scand J Rheumatol. 2003; 32: 312-4.

9. Rigante D, Ansuini V, Caldarelli M, et al. Hydrocephalus in CINCA syndrome treated with anakinra. Childs Nerv Syst. 2006; 22: 334-7.

10. Rigante D, Leone A, Marrocco R, et al. Longterm response after 6-year treatment with anakinra and onset of focal bone erosion in neonatal-onset multisystem inflammatory disease (NOMID/CINCA). Rheumatol Int. 2011; 31: 1661-4.

11. Rigante D, Verrecchia E, Falsini B, Manna R. Switch from anakinra to canakinumab in a severe case of CINCA syndrome. Int J Rheum Dis. 2016; 19: 1354-6.

12. Lopalco G, Cantarini L, Vitale A, et al. Interleukin-1 as a common denominator from autoinflammatory to autoimmune disorders: premises, perils, and perspectives. Mediators Inflamm. 2015; 2015: 194864.

13. Cantarini L, Iacoponi F, Lucherini OM, et al. Validation of a diagnostic score for the diagnosis of autoinflammatory diseases in adults. Int $\mathrm{J}$ Immunopathol Pharmacol. 2011; 24: 695-702.

14. ter Haar NM, Annink KV, Al-Mayouf SM, et al. Development of the autoinflammatory disease damage index (ADDI). Ann Rheum Dis. 2017; 76: 821-30.

15. Kuemmerle-Deschner JB, Ozen S, Tyrrell PN, et al. Diagnostic criteria for cryopyrin-associated periodic syndrome (CAPS). Ann Rheum Dis. 2017; 76: 942-7.

16. Maloney E, Stanescu AL, Ngo AV, et al. The pediatric patella: normal development, anatomical variants and malformations, stability, imaging, and injury patterns. Semin Musculoskelet Radiol 2018; 22: 81-94. 